\title{
Relationship between antibiotic resistance and sickle cell anemia: preliminary evidence from a pediatric carriage study in Ghana
}

This article was published in the following Dove Press journal:

Infection and Drug Resistance

26 July 2013

Number of times this article has been viewed

\author{
Eric S Donkor' \\ Ebenezer Foster-Nyarko² \\ Christabel C Enweronu- \\ Laryea $^{3}$ \\ 'Department of Microbiology, \\ University of Ghana Medical School, \\ Accra, Ghana; ${ }^{2}$ Department of Medical \\ Laboratory Science, School of Allied \\ Health Sciences, University of Ghana, \\ Accra, Ghana; ${ }^{3}$ Department of Child \\ Health, University of Ghana Medical \\ School, Accra, Ghana
}

Correspondence: Eric S Donkor Department of Microbiology, University of Ghana Medical School, PO Box 4236, Accra, Ghana

Tel +233 302665404

Email ericsdon@hotmail.com
Background: Antibiotics are frequently used among people with sickle cell anemia (homozygous SS or HbSS disease), especially for prophylaxis. However, the relationship between antibiotic resistance and people with HbSS disease has not been adequately studied, especially in the developing world. The objectives of the study were (1) to compare antibiotic resistance patterns of nasal Staphylococcus aureus between children with HbSS disease and children without HbSS disease (healthy children) and (2) to evaluate nasopharyngeal carriage of antibiotic-resistant Streptococcus pneumoniae among children with HbSS disease.

Methods: This was a prospective cross-sectional study, and the subjects were children under 12 years old. Nasal swabs were collected from 50 children with HbSS disease and 50 children without HbSS disease. Nasopharyngeal swabs were collected from another group of 92 children with HbSS disease. The nasal and nasopharyngeal swabs were cultured for $S$. aureus and $S$. pneumoniae, respectively. Susceptibility testing was carried out on the $S$. aureus and S. pneumoniae isolates for various antibiotics, including penicillin, ampicillin, cefuroxime, erythromycin, cloxacillin, and cotrimoxazole.

Results: The carriage rates of $S$. aureus among pediatric subjects with HbSS disease and those without $\mathrm{HbSS}$ disease were $48 \%$ and $50 \%$, respectively $(P>0.05)$. S . pneumoniae carriage among the pediatric subjects with $\mathrm{HbSS}$ disease was $10 \%$. Antibiotic resistance patterns of $S$. aureus carried by children with HbSS disease and children without HbSS disease were similar, and the $S$. aureus resistance rates were $>40 \%$ for the various antibiotics, with the exception of erythromycin and cloxacillin. Low levels of $S$. pneumoniae resistance $(0 \%-11 \%)$ were observed for the various antibiotics tested except cotrimoxazole, which showed an extremely high-percentage resistance (100\%).

Conclusion: Sickling status is not a risk factor for carriage of $S$. aureus. In this cohort of Ghanaian children with HbSS disease, S. aureus is higher in carriage and more antibioticresistant, compared to $S$. pneumoniae.

Keywords: Paediatric, sickle cell anaemia, antibiotic resistance, Ghana

\section{Introduction}

Antibiotic resistance has been attributed to the misuse of antibiotics, which exert selective pressure favoring the emergence of resistant strains. ${ }^{1,2}$ Antibiotic use selects for resistance not only in pathogenic bacteria, but also in the commensal flora of exposed individuals. Studies of the prevalence of antibiotic resistance in commensal microflora are vital in monitoring and understanding the process of antimicrobial-mediated selection in a population. ${ }^{3,4}$ Clinically, antibiotic resistance in commensal microflora is of significance because such organisms could cause opportunistic infections, as in immunosupression or in underlying diseases such as sickle cell disease. ${ }^{5}$ 
Antibiotics are frequently used among people with homozygous SS (HbSS) disease, especially for prophylaxis. However, the relationship between antibiotic resistance and people with HbSS disease has not been adequately studied, especially in sub-Saharan Africa. In Ghana, one study investigated Streptococcus pneumoniae penicillin resistance among people with HbSS disease. ${ }^{6}$ However, the study did not include a control arm, which makes it difficult to deduce any trend in antibiotic resistance that may be peculiar to people with HbSS disease. Recently, a Ugandan study showed that S. pneumoniae carried by people with HbSS disease were highly resistant to penicillin and cotrimoxazole, compared to a control group, but no significant variations were observed for chloramphenicol, erythromycin, cefriaxone, rifampin, or perfloxacin. ${ }^{7}$ Carriage studies that have investigated antibiotic resistance among people with HbSS disease in subSaharan Africa seem to have focused on S. pneumoniae, ${ }^{6-8}$ which is probably due to the prominence of the organism in bacteraemia in HbSS disease in Africa. However, some studies indicate that Staphylococcus aureus could be more important in invasive disease among people with HbSS disease than $S$. pneumoniae. ${ }^{9-13}$ So far, very few studies have investigated the antibiotic resistance of $S$. aureus carried by people with HbSS disease in sub-Saharan Africa, probably because there are few reports of $S$. aureus-related clinical diseases in children with HbSS in the region. Such a study would contribute to our understanding of the epidemiology of $S$. aureus diseases among people with HbSS disease in the region, and allow improvements in antimicrobial therapy for such infections.

In this study, we present preliminary carriage data on S. aureus and S. pneumoniae among children with $\mathrm{HbSS}$ disease in Ghana. The objectives of the study were (1) to compare antibiotic resistance patterns of nasal $S$. aureus between children with $\mathrm{HbSS}$ disease and children without HbSS disease (healthy children) and (2) to evaluate nasopharyngeal carriage of antibiotic-resistant $S$. pneumoniae among children with HbSS disease.

\section{Material and methods}

\section{Study area and sampling}

The study was carried out at the pediatric unit of Korle-Bu Teaching Hospital in Accra, the capital city of Ghana, from February 2006 to April 2007. Korle-Bu Teaching Hospital is the largest hospital in Ghana, and the pediatric unit has a sickle cell clinic that runs once a week. The study was a cross-sectional study of randomly selected pediatric subjects under 12 years who were attending the hospital for reviews or registration for the first time. Based on $95 \%$ confidence limits with an allowable error of $10 \%, 100$ subjects, including 50 who had HbSS disease and 50 without HbSS disease (healthy children), took part in the $S$. aureus carriage study, while a second group of 92 children who had HbSS disease took part in the S. pneumoniae carriage study. The diagnosis of HbSS was based on hemoglobin electrophoresis. In the S. aureus carriage study, nasal specimens were collected from the study participants, while nasopharyngeal specimens were collected from the study participants in the S. pneumoniae carriage study. The specimens were collected with the aid of swabs and processed immediately in the laboratory.

\section{Bacteria isolation and antibiotic susceptibility testing}

The swab specimens were cultured for $S$. aureus and S. pneumoniae using standard microbiological methods. ${ }^{14}$ The specimens were inoculated onto plates of blood agar (Oxoid Ltd, Basingstoke, UK) and incubated at $37^{\circ} \mathrm{C}$ for 18-24 hours. Plates for $S$. aureus were incubated aerobically, while plates for S. pneumoniae were incubated in 5\% $\mathrm{CO}_{2}{ }^{14,15}$ Suspected $S$. aureus isolates were confirmed using the coagulase test, ${ }^{14}$ while suspected $S$. pneumoniae isolates were confirmed using the optochin test ${ }^{14,16}$ The confirmed isolates of $S$. aureus and $S$. pneumoniae were purified and subjected to antibiotic susceptibility testing using a modified form of the Kirby Bauer method. ${ }^{17,18}$ Susceptibility testing of $S$. pneumoniae and $S$. aureus isolates was done for penicillin, ampicillin, cefuroxime, erythromycin, cloxacillin, and cotrimoxazole (Oxoid Ltd, Basingstoke, UK). These antibiotics are commonly used in Ghana for treating bacterial infections, including those caused by $S$. aureus and $S$. pneumoniae. The antibiotic susceptibility-testing procedure employed for the bacteria isolates was as follows. On the agar plate of the test organism, a sterile loop was used to touch four to five morphologically similar colonies and inoculate $5 \mathrm{~mL}$ sterile saline water until the turbidity was comparable to $0.5 \%$ McFarland's standard. A loopful of the suspension was aseptically transferred to the center of a Mueller-Hinton agar plate (Oxoid Ltd), and a sterile cotton swab was used to streak the entire surface of the plate. The moisture was allowed to be absorbed for at least 15 minutes, and sterile forceps were used to apply the antibiotic discs to the surface of the agar plate while ensuring the discs were not placed less than $24 \mathrm{~mm}$ apart. The plates were incubated at $37^{\circ} \mathrm{C}$ for 18-24 hours, after which a ruler and a pair of vernier calipers were used to measure the zones of inhibition around the antibiotic discs. The results were recorded. S. aureus NCTC 
6571 and S. pneumoniae NCTC 10319 were used as controls for the $S$. aureus and $S$. pneumoniae isolates, respectively. The minimum cutoff point for an $S$. aureus or S. pneumoniae isolate to be classified as sensitive andor resistant was based on the NCLS break-point system.

The study protocol was approved by the Ethical and Protocol Review Committees of the School of Allied Health Sciences (University of Ghana) and the University of Ghana Medical School, and consent was obtained from parents or guardians of the children recruited for the study.

\section{Data analysis}

The data collected in the study were entered into MS Excel and analyzed in STATA 7.0 (Strata Corp, College Station, TX, USA). Descriptive analysis was carried out on the study variables, and prevalence rates were reported as percentages and $95 \%$ confidence intervals. Chi-square was used to evaluate significant associations among the study variables, and $P$ values of $<0.05$ were considered statistically significant.

\section{Results and discussion}

Epidemiological data collected in this study, including sampling, demographic information, and bacteria carriage rates, are summarized in Table 1 . The mean age of subjects recruited in the $S$. aureus carriage study was 3.5 years for those with HbSS disease and 2.5 years for those without $\mathrm{HbSS}$ disease (control group). The carriage rates of $S$. aureus among people with $\mathrm{HbSS}$ disease and people without $\mathrm{HbSS}$ disease were 48\% (95\% confidence interval (CI): 34.2-61.9), and 50\% (95\% CI: 36.2-63.9), respectively. There was no significant difference in $S$. aureus carriage between the two populations $(P<0.05)$. The anterior nares are the most consistent site of staphylococcal colonization, and carriage

Table I Features of Staphylococcus aureus and Streptococcus pneumoniae carriage studies

\begin{tabular}{lll}
\hline Parameter & S. aureus study & S. pneumoniae study \\
\hline Type of specimen used & Nasal swabs & Nasopharyngeal swabs \\
Number HbSS+ children & 50 & 92 \\
$\begin{array}{l}\text { Number HbSS- children } \\
\text { (control group) }\end{array}$ & 50 & No subjects recruited \\
Gender distribution & 58 males, & 54 males, \\
Mean age of subjects & 34 females & 46 females \\
Carriage among & 3 years ${ }^{\mathrm{a}}$ & 8 years \\
HbSS+ children & $48 \%$ & $10 \%$ \\
Carriage among & & \\
HbSS- children & $50 \%$ & $\mathrm{n} / \mathrm{a}$
\end{tabular}

Note: ${ }^{\mathrm{M}}$ Mean ages of HbSS+ children and HbSS- children (control group) were 3.5 years and 2.5 years, respectively.

Abbreviation: HbSS, homozygous SS disease. is more common in children than in adults, ${ }^{19-21}$ hence the use of nasal specimens from pediatric subjects in this study. The trend of $S$. aureus carriage in this study concurs with a study done in Gabon, which reported an $S$. aureus carriage rate of 46.6\% among children with $\mathrm{HbSS}$ disease and 46.9\% among children without HbSS disease. ${ }^{18}$ About $75 \%$ of people with $\mathrm{HbSS}$ disease are in sub-Saharan Africa, ${ }^{22}$ and evidence from our study and from that carried out in Gabon ${ }^{8}$ indicates that sickling status is not a risk factor for $S$. aureus carriage. In the developed world, there are hardly any data on carriage of $S$. aureus among people with $\mathrm{HbSS}$ disease, though several studies have investigated carriage of the organism in the general population in some of these countries. ${ }^{23-25}$ In the USA, two studies carried out on children younger than 7 years reported $S$. aureus carriage rates of $9 \%{ }^{23}$ and $14 \% .^{24}$ In Portugal, a study reported $S$. aureus carriage of $17 \%$ among children younger than 6 years. ${ }^{25}$ These prevalence rates most probably apply to children with $\mathrm{HbSS}$ disease in the respective countries, as our data confirm that sickling status is not a risk factor for carriage of $S$. aureus. Thus, it is likely that $S$. aureus carriage among children with $\mathrm{HbSS}$ disease in sub-Saharan African countries such as Ghana is significantly higher than among their counterparts in the developed world, such as in the USA.

The mean age of subjects recruited in the S. pneumoniae carriage study was 8 years, and the carriage rate of S. pneumoniae was 10\% (95\% CI: 3.9-16.1). Carriage rates of $S$. pneumoniae among children with $\mathrm{HbSS}$ disease reported in other countries were $33 \%$ in Uganda, ${ }^{7} 14 \%$ in Gabon, ${ }^{8}$ $13 \%$ in the USA, ${ }^{26}$ and $21 \%$ in the UK. ${ }^{27}$ S. pneumoniae carriage among subjects with $\mathrm{HbSS}$ disease in the current study $(10 \%)$ is similar to a carriage rate of $16 \%$ previously reported among HbSS disease children in Ghana, ${ }^{6}$ but significantly lower than most of the carriage rates $(>40 \%)$ that have been reported generally among children in Ghana. ${ }^{28,29}$ The relatively lower carriage rate of $S$. pneumoniae among pediatric subjects with HbSS disease in Ghana, which has also been reported in $\mathrm{Uganda}^{7}$ and in some western countries such as the USA, ${ }^{20}$ is probably due to the routine prophylactic use of penicillin among people with $\mathrm{HbSS}$ disease. Contrary to the disparity of $S$. pneumoniae carriage between people with and without HbSS disease in Ghana (as described above), we did not observe a relatively lower carriage rate of $S$. aureus among people with $\mathrm{HbSS}$ disease, probably because unlike $S$. pneumoniae, $S$. aureus is highly resistant to penicillin because of its production of beta-lactamase. ${ }^{21}$

As shown in Figure 1, the trend of resistance of the S. aureus isolates from pediatric subjects with $\mathrm{HbSS}$ 


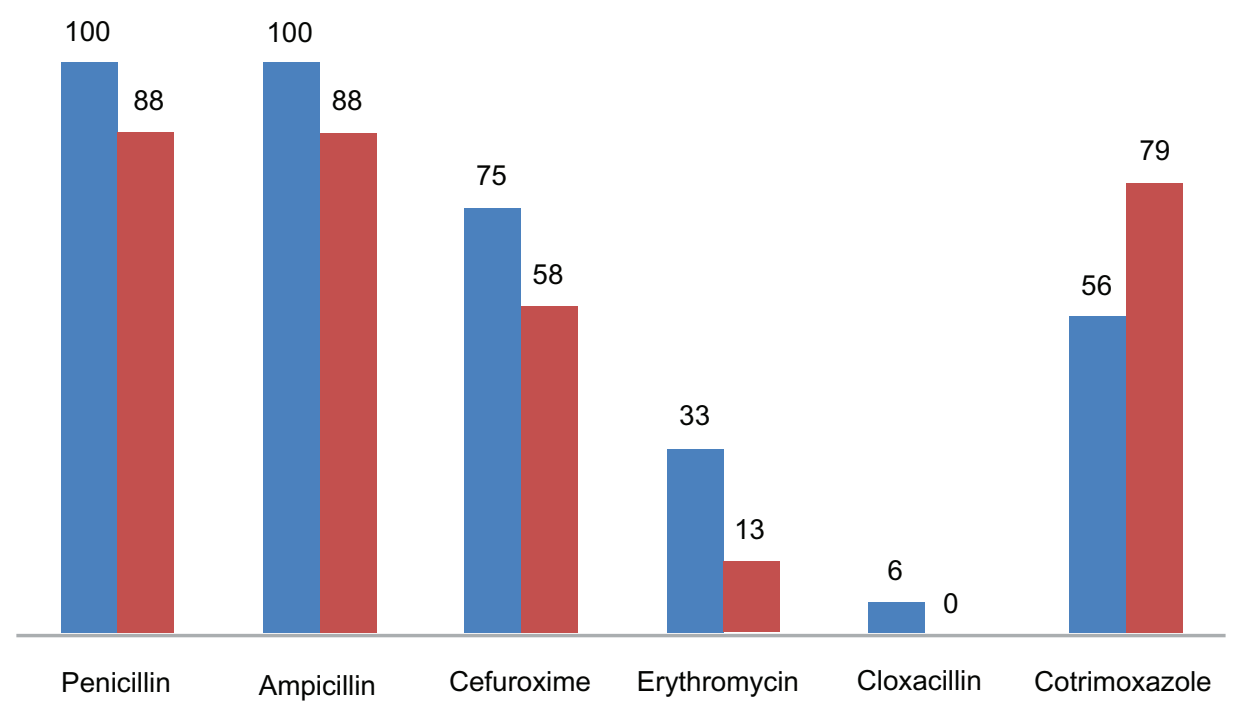

Figure I Antibiotic resistance of Staphylococcus aureus isolated from $\mathrm{HbSS}+$ and $\mathrm{HbSS}-$ children.

Notes: The pattern of resistance of $\mathrm{S}$. aureus isolates from $\mathrm{HbSS}+$ and $\mathrm{HbSS}-$ children were similar and did not show any significant differences at $P<0.05$. For both $\mathrm{HbSS}+$ and HbSS- children, resistance was highest for the penicillin/ampicillin and lowest for cloxacillin.

Abbreviation: HbSS, homozygous SS disease.

disease and those without HbSS disease were similar and did not show any significant differences for all six of the antibiotics tested. By comparison, a study among Gabonese children did not show significant differences in antibiotic resistance between subjects with HbSS disease and those without HbSS disease for various encapsulated organisms, including $S$. aureus, S. pneumoniae, and Haemophilus influenzae. ${ }^{8}$ By contrast, a Ugandan study showed that $S$. pneumoniae carried by people with $\mathrm{HbSS}$ disease were highly resistant to penicillin and cotrimoxazole, compared to people without HbSS disease. ${ }^{7}$ This shows that the relationship between antibiotic resistance and people with HbSS disease may vary according to geographical setting, which is likely to be related to the epidemiology of antibiotic usage in a particular geographic setting. In Ghana, there is a high rate of prescriptions for antibiotics for people with HbSS disease, which may be offset by a high rate of irrational use of antibiotics, that is, through self-medication, in the general Ghanaian community. ${ }^{30,31}$ This may explain the absence of differences in resistance rates between $S$. aureus isolates from people with $\mathrm{HbSS}$ disease and those without HbSS disease in this study. In the USA, where self-medication is relatively uncommon and antibiotics such as penicillin are routinely used as prophylaxis for HbSS disease, several studies showed that S. pneumoniae isolated from people with HbSS disease had a significantly higher prevalence of penicillin resis- tance, compared to isolates from people without $\mathrm{HbSS}$ disease. ${ }^{32,33}$

The antibiotic-resistance pattern of $S$. pneumoniae isolated from pediatric subjects with $\mathrm{HbSS}$ disease is reported in Figure 1, and shows a very low prevalence of resistance for most of the antibiotics tested. The antibiotic-susceptibility pattern for the $S$. pneumoniae isolates is similar to that reported by a recent pneumococcal carriage study carried out generally among children in the study area. ${ }^{29}$ Of interest is penicillin resistance, as the antibiotic is commonly used in pneumococcal prophylaxis among people with $\mathrm{HbSS}$ in Ghana. While in this study pneumococcal penicillin resistance among people with $\mathrm{HbSS}$ was nil, a similar study found a prevalence rate of $40 \%$ in another region of Ghana, ${ }^{6}$ suggesting wide variations in pneumococcal penicillin resistance in different parts of the country. The prevalence of penicillin-resistant $S$. pneumoniae among people with HbSS disease reported recently in other sub-Saharan African countries is $100 \%$ in Uganda $^{7}$ and $30 \%$ in Gabon. ${ }^{8}$ These data further suggest wide variations in pneumococcal penicillin resistance in sub-Saharan Africa with respect to people with HbSS disease and to the potential for such patients to carry extremely high levels of penicillin resistance. Though we did not observe pneumococcal penicillin resistance, we predict a significant increase among people with $\mathrm{HbSS}$ disease in Ghana, similar to the high rates reported in other sub-Saharan African countries, due to the similarity in distribution of 


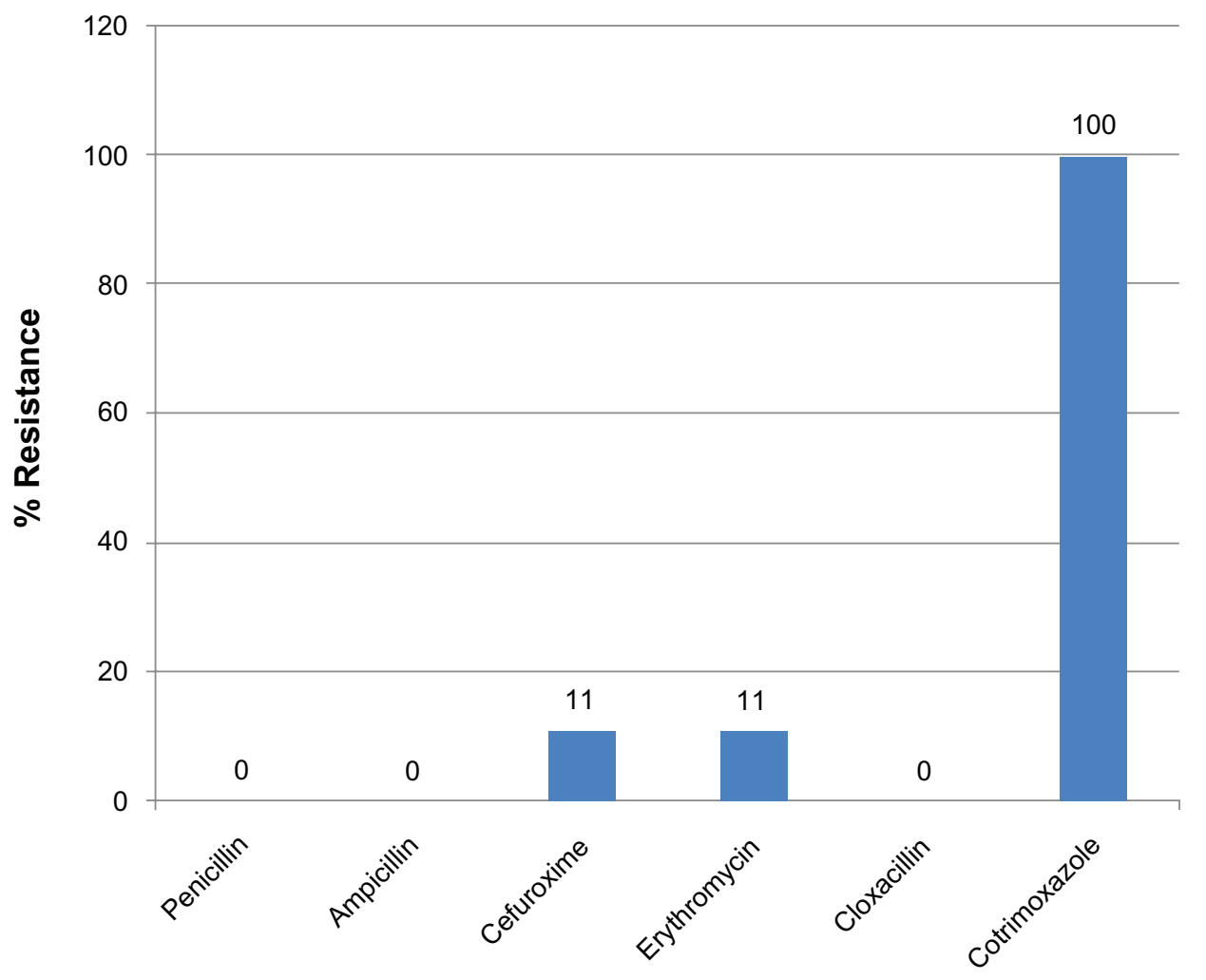

Figure 2 Antibiotic \% resistance of Streptococcus pneumoniae isolated from HbSS+ children.

Note: Low levels of percentage resistance $(0 \%-11 \%)$ were observed for the various antibiotics tested except cotrimoxazole, which showed an extremely high prevalence of $\%$ resistance (100\%).

Abbreviation: HbSS, homozygous SS disease.

antibiotic-resistance risk factors in sub-Saharan Africa. ${ }^{34}$ The increasing trend of penicillin-resistant $S$. pneumoniae strains in relation to people with $\mathrm{HbSS}$ disease in sub-Saharan Africa is a cause for concern, as this is likely to reduce the prophylactic ability of the antibiotic among such patients. Fortunately, $S$. pneumoniae conjugate vaccines that have proven effective in children are currently available for use in both the developed and developing world. An excellent feature of such conjugate vaccines is their ability to eliminate carriage of $S$. pneumoniae and hence reduce invasive pneumococcal disease. ${ }^{35}$ The vaccines, however, do not offer a complete solution to the problem of invasive pneumococcal disease among children, as they cover only 7-13 serotypes of the 94 known pneumococcal serotypes. ${ }^{35}$ It is worth noting that pneumococcal resistance to cotrimoxazole has reached its peak in Ghana, and the antibiotic is most probably useless for treating any pneumococcal infections in the country.

In Ghana, there are no surveillance data on microbial diseases in people with HbSS disease. However, there are some data from a few African countries that depict an interesting isolation pattern of causative agents. In a Ugandan study involving $155 \mathrm{HbSS}$ patients, the important etio- logical agents were S. aureus (60\%), H. influenzae (19\%) and Staphylococcus epidermidis (9\%). ${ }^{9}$ A Kenyan study involving 108 children with $\mathrm{HbSS}$ disease identified the most common causes of bacteraemia as S. pneumoniae (41\%), non-typhi Salmonella species (18\%), and H. influenzae type b $(12 \%){ }^{36}$ In Nigeria, five studies ${ }^{10-12,37,38}$ have investigated bacteraemia among 57 to 304 children with HbSS disease: the most prevalent etiological agent in these studies varied, and included S. aureus (44\%), S. pneumoniae (40\%), Salmonellae spp. (26\%), and Klebsiella spp. (which had prevalence rates of $32 \%$ and $26 \%$ in two different studies). S. pneumoniae is probably regarded as the most important cause of invasive bacterial disease among people with $\mathrm{HbSS}$ disease, as shown by the institution of penicillin prophylaxis and vaccination for people with HbSS disease. However, the above data from sub-Saharan Africa indicate that other bacterial agents, especially $S$. aureus, may supersede $S$. pneumoniae as invasive diseases among people with $\mathrm{HbSS}$, which partly agrees with our data showing a five-fold higher carriage rate of $S$. aureus (48\%) compared to $S$. pneumoniae (10\%). It is important to put the findings of the current study in the context of pneumococcal vaccination, which is currently 
underway in many African countries. S. pneumoniae carriage is known to be inversely associated with $S$. aureus carriage in children, ${ }^{39}$ which we could not investigate in our sample, as the $S$. aureus and $S$. pneumoniae carriage studies were carried out among different study subjects. This implies that massive pneumococcal vaccination underway in sub-Saharan Africa may further cause an upward shift in $S$. aureus carriage, and consequently, an increase in the burden of $S$. aureus diseases, which would be disturbing, given the emergence of methicillin-resistant $S$. aureus. ${ }^{40,41}$ Such an evolutionary process is expected to cause a greater burden among $\mathrm{HbSS}$ patients who are more susceptible to bacterial infections. In view of this, there is the need for further antibiotic susceptibility-testing studies on $S$. aureus in sub-Saharan Africa to improve empirical antibiotic treatment of $S$. aureus diseases among HbSS patients in the region.

\section{Conclusion}

This study provides some of the first data on carriage of $S$. aureus among people with HbSS disease in sub-Saharan Africa, and it indicates that sickling status is not a risk factor for carriage of $S$. aureus. The relatively high carriage of S. aureus among people with HbSS disease in sub-Saharan African countries such as Ghana may explain the predominance of the organism in invasive disease among people with HbSS disease in sub-Saharan Africa. Antibiotic-resistance patterns of $S$. aureus carried by people with $\mathrm{HbSS}$ disease and people without HbSS disease in Ghana are similar, and the $S$. aureus resistance rates are high. Carriage of antibioticresistant $S$. pneumoniae among the children with HbSS disease investigated in this study is quite low; however, there is a high potential for rapid increase in resistance, as observed in some sub-Saharan African areas.

The main limitations of this study are that we did not have a control arm for the carriage study on S. pneumoniae. Our data are preliminary, and do not cover aspects of antibiotic exposure or subtyping/serotyping of the $S$. aureus and $S$. pneumoniae that were used for investigating antibiotic resistance. A further study on the subject in these areas is desirable.

\section{Disclosure}

The authors report no conflicts of interest in this work.

\section{References}

1. Alanis AJ. Resistance to antibiotics: are we in the post-antibiotic era? Arch Med Res. 2005;36:697-705.

2. Van de Bogaard AE, Stobberingh EE. Epidemiology of resistance to antibiotics. Links between animals and humans. Int JAntimicrob Agents. 2000;14:327-335.
3. Caprioli A, Busani L, Martel JL, Helmuth R. Monitoring of antibiotic resistance in bacteria of animal origin: epidemiological and microbiological methodologies. Int J Antimicrob Agents. 2000;14:295-301.

4. Andremont A. Commensal flora may play key role in spreading antibiotic resistance. ASM News. 2003;69:601-607.

5. Booth C, Inusa B, Obaro SK. Infection in sickle cell disease: a review. Int J Infect Dis. 2009;14:2-12.

6. Baffoe-Bonnie B, Adu-Sarkodie Y, Twumasi P, Akoto YO. Antimicrobial susceptibility of pneumococci colonizing the nasopharynx of children with sickle cell disease. Ghana Med J. 2000;34:18-20.

7. Kateete DP, Kajumbula H, Kaddu-Mulindwa DH, Ssevviri AK. Nasopharyngeal carriage rate of Streptococcus pneumoniae in Ugandan children with sickle cell disease. BMC Res Notes. 2012;5:28.

8. Schaumburg F, Biallas B, Feugap EN, et al. Carriage of encapsulated bacteria in Gabonese children with sickle cell anaemia. Clin Microbiol Infect. 2013;19(3):235-241.

9. Kizito E, Mworozi E, Ndugwa C, Serjeant GR. Bacteraemia in homozygous sickle cell disease in Africa: is pneumococcal prophylaxis justified? Arch Dis Child. 2007;92:21-23.

10. Akuse RM. Variation in the pattern of bacterial infection in patients with sickle cell disease requiring admission. J Trop Pediatr. 1996;42: 318-323.

11. Okuonghae HO, Nwankwo MU, Offor EC. Pattern of bacteremia in febrile children with sickle cell anemia. Ann Trop Pediatr. 1993;13: $55-64$.

12. Aken'ova YA, Bakare RA, Okunade MA. Septicaemia in sickle cell anaemia patients: the Ibadan experience. Cent Afri J Med. 1998;44: $102-104$.

13. Thanni LO. Bacterial osteomyelitis in major sickling haemoglobinopathies: geographic difference in pathogen prevalence. Afr Health Sci. 2006;6(4):236-239.

14. Baron JE, Peterson LR, Finegold SM. Bailey and Scott Diagnostic Microbiology. 9th ed. Maryland Heights, MO: C V Mosby Co; 1994.

15. Austrian R, Collins P. Importance of carbon dioxide in the isolation of pneumococci. J Bacteriol. 1966;92:1281-1284.

16. Bowers EF, Jeffries LR. Optochin in the identification of str. pneumoniae. J Clin Pathol. 1955;8:58-60.

17. Bauer AW, Kirby WM, Sherris JC, Turck M. Antibiotic susceptibility testing by a standardized single disk method. Am J Clin Pathol. 1966;45(4):493-496.

18. National Committee for Clinical Laboratory Standards, 2005. Performance standards for antimicrobial susceptibility testing. Fifteenth informational supplement. NCCLS document M100-S15. Wayne, PA, USA. Available from: http://www.clsi.org/. Accessed November 6, 2012.

19. Kluytmans J, van Belkum A, Verbrugh H. Nasal carriage of Staphylococcus aureus: epidemiology, underlying mechanisms, and associated risks. Clin Microbiol Rev. 1997;10:505-220.

20. Armstrong-Esther CA, Smith JE. Carriage patterns of Staphylococcus aureus in a healthy non-hospital population of adults and children. Ann Hum Biol. 1976;3:221-227.

21. Wertheim HF, Melles DC, Vos MC, et al. The role of nasal carriage in Staphylococcus aureus infections. Lancet Infect Dis. 2005;5:751-762.

22. World Health Organization. Sickle-cell anaemia - Report by the Secretariat. World Health Organization, Geneva; 2006. Accessed November 27, 2010.

23. Cheng-Immergluck L, Kanungo S, Schwartz A, McIntyre A, Schreckenberger PC, Diaz PS. Prevalence of Streptococcus pneumoniae and Staphylococcus aureus nasopharyngeal colonization in healthy children in the United States. Epidemiol and Infect. 2004;132(2): $159-166$.

24. Lee GM, Huang SS, Rifas-Shiman SL, Hinrichsen VL, et al. Epidemiology and risk factors for Staphylococcus aureus colonization in children in the post-PCV7 era. BMC Infect Dis. 2009;9:110.

25. Tavares DA, Sá-Leão R, Miragaia M, de Lencastre H. Large screening of CA-MRSA among Staphylococcus aureus colonizing healthy young children living in two areas (urban and rural) of Portugal. BMC Infect Dis. 2010;10:110. 
26. Daw NC, Wilimas JA, Wang WC, et al. Nasopharyngeal carriage of penicillin-resistant Streptococcus pneumoniae in children with sickle cell disease. Pediatrics. 1997;99(4):E7.

27. Alexander E, Telfer P, Rashid H, Ali KA, Booy R. Nasopharyngeal carriage rate of Streptococcus pneumoniae in children with sickle cell disease before and after the introduction of heptavalent pneumococcal conjugate vaccine. J Infect Public Health. 2008;1(1):40-44.

28. Denno DM, Frimpong E, Gregory M, Steele RW. Nasopharyngeal carriage and susceptibility patterns of Streptococcus pneumoniae in Kumasi, Ghana. West Afr J Med. 2002;21:233-236.

29. Arhin R. Penicillin resistant Streptococcus pneumoniae in the Nasopharynx of Children under Six Years [master's thesis]. Accra: University of Ghana; 2012.

30. Adu-Sarkodie YA. Antimicrobial self medication in patients attending a sexually transmitted diseases clinic. Int J STD AIDS. 1997;8:456-458.

31. Donkor ES, Tetteh-Quarcoo PB, Nartey P, Agyeman IO. Selfmedication practices with antibiotics among tertiary level students in Accra, Ghana: a cross-sectional study. Int J Environ Res Public Health. 2012;9:3519-3529.

32. Anglin DL, Siegel JD, Pacini DL, Smith SJ, Adams G, Buchanan GR. Effect of penicillin prophylaxis on nasopharyngeal colonization with Streptococcus pneumoniae in children with sickle cell anemia. JPediatr. 1984;104:18-22.

33. Steele RW, Warrier R, Unkel PJ, et al. Colonization with antibioticresistant Streptococcus pneumoniae in children with sickle cell disease. J Pediatr. 1996;128:531-535.
34. Kimanj'a AN. A situational analysis of antimicrobial drug resistance in Africa: are we losing the battle? Ethiop J Health Sci. 2012;22(2): 135-143.

35. World Health Organization. Pneumococcal conjugate vaccine for childhood immunization - WHO position paper. Wkly Epidemiol Rec. 2007;82:93-104.

36. Williams TN, Uyoga S, Macharia A, et al. Bacteraemia in Kenyan children with sickle-cell anaemia: a retrospective cohort and case-control study. Lancet. 2009;374:1364-1370.

37. Akinyanju O, Johnson AO. Acute illness in Nigerian children with sickle cell anemia. Ann Trop Paediatr. 1987;7:181-186.

38. Maharajan R, Flemming AF, Egler L. Pattern of infections among patients with sickle cell anaemia requiring hospital admission. Nig J of Paed. 1983;10:13-17.

39. Lebon A, Verkaik NJ, de Vogel CP, et al. The inverse correlation between Staphylococcus aureus and Streptococcus pneumoniae colonization in infants is not explained by differences in serum antibody levels in the Generation R Study. Clin Vac Immunol. 2011;18:180-183.

40. David MZ, Daum RS. Community-associated methicillin resistant Staphylococcus aureus: epidemiology and clinical consequences of an emerging epidemic. Clin Microbiol Rev. 2010;23:616-687.

41. Rehm SJ, Tice A. Staphylococcus aureus: Methicillin-susceptible $S$. aureus to methicillin-resistant $S$. aureus and vancomycin-resistant S. aureus. Clin Infect Dis. 2010;51:176-182.
Infection and Drug Resistance

\section{Publish your work in this journal}

Infection and Drug Resistance is an international, peer-reviewed openaccess journal that focuses on the optimal treatment of infection (bacterial, fungal and viral) and the development and institution of preventive strategies to minimize the development and spread of resistance. The journal is specifically concerned with the epidemiology of antibiotic

\section{Dovepress}

resistance and the mechanisms of resistance development and diffusion in both hospitals and the community. The manuscript management system is completely online and includes a very quick and fair peerreview system, which is all easy to use. Visit http://www.dovepress.com/ testimonials.php to read real quotes from published authors. 